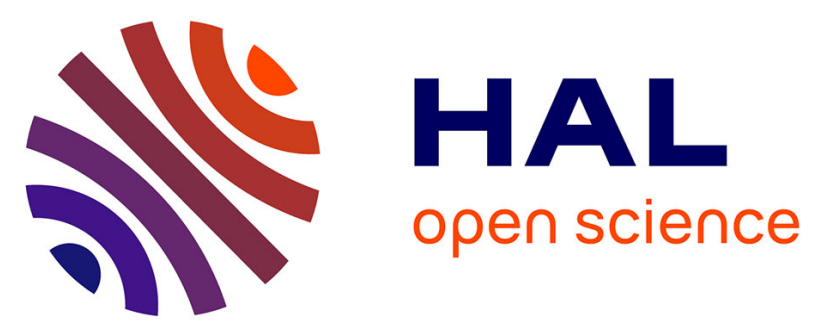

\title{
Comparison of Keller-Miksis model and finite element bubble dynamics simulations in a confined medium. Application to the hydrodynamic ram
}

Thomas Fourest, Éric Deletombe, Vincent Faucher, M. Arrigoni, Jacques

Dupas, Jean-Marc Laurens

\section{To cite this version:}

Thomas Fourest, Éric Deletombe, Vincent Faucher, M. Arrigoni, Jacques Dupas, et al.. Comparison of Keller-Miksis model and finite element bubble dynamics simulations in a confined medium. Application to the hydrodynamic ram. European Journal of Mechanics - B/Fluids, 2018, 68, pp.66-75. 10.1016/j.euromechflu.2017.11.004 . hal-01664736

\section{HAL Id: hal-01664736 https://hal.science/hal-01664736}

Submitted on 22 Nov 2021

HAL is a multi-disciplinary open access archive for the deposit and dissemination of scientific research documents, whether they are published or not. The documents may come from teaching and research institutions in France or abroad, or from public or private research centers.
L'archive ouverte pluridisciplinaire HAL, est destinée au dépôt et à la diffusion de documents scientifiques de niveau recherche, publiés ou non, émanant des établissements d'enseignement et de recherche français ou étrangers, des laboratoires publics ou privés.

\section{(c) (1) $\$$}

Distributed under a Creative Commons Attribution - NonCommerciall 4.0 International 


\title{
Comparison of Keller-Miksis model and finite element bubble dynamics simulations in a confined medium. Application to the Hydrodynamic Ram
}

\author{
Thomas Fourest ${ }^{\mathrm{a}, *}$, Eric Deletombe $^{\mathrm{a}}$, Vincent Faucher ${ }^{\mathrm{b}}$, Michel Arrigoni ${ }^{\mathrm{c}}$, \\ Jacques Dupas ${ }^{a}$, Jean-Marc Laurens ${ }^{c}$ \\ a ONERA - The French Aerospace Lab, F-59045, Lille, France \\ b CEA, DEN, DANS, DM2S, SEMT, DYN, Gif sur Yvette F-91191, France \\ c ENSTA-Bretagne, FRE CNRS 3744 - IRDL, F-29806, Brest, France
}

\begin{abstract}
Hydrodynamic ram (HRAM) is a phenomenon that occurs when a high-speed/high energy projectile penetrates a liquid-filled container. The container must adapt to allow the variation of volume of the cavity created, that generates a large loading due to the liquid momentum. This complex fluid-structure interaction phenomenon has been identified as one of the major threat to aircraft safety. An approach based on bubble dynamics models has been developed by the authors to take this phenomenon into account when designing fuel tanks. In the present work, the authors compare ALE finite element simulations using EUROPLEXUS to a modification of the Keller-Miksis model to account for the confinement effect of the container. In the studied domain, the agreement on the cases simulated is found quite acceptable: $3 \%$ and $1 \%$ differences respectively in amplitude and period for the bubble dynamic and less than $1 \%$ for the impulse transmitted to the structure.
\end{abstract}

\section{Introduction}

A large amount of research has been performed over the last decades on the problem of cavitation and bubble dynamics. However very few studies deal with the problem of cavitation in confined fluids. This problem has practical applications, one of them being the Hydrodynamic Ram (HRAM) phenomenon. This phenomenon occurs when a high-speed/high energy projectile penetrates a liquid-filled container [1-3]. The projectile creates in its wake a cavity and transfers its momentum to the surrounding liquid. An important difference between HRAM and other cavitation problem is that the cause of damage is not the same, it is the bursting of the container due to the solicitation generated by the growth of a large bubble in a closed container: the container may also experience a large deformation in order to accommodate the gas bubble expansion. Unlike in other cavitation applications, in HRAM events the container is usually large enough, with enough distance between the bubble and the walls: no hydrodynamic jet is formed towards the walls as in most cavitation applications [4-6]. The container must then adapt to allow the variation of volume of this cavity, which generates a large transient load due

\footnotetext{
* Corresponding author.

E-mail address: thomas.fourest@onera.fr (T. Fourest).
}

to liquid momentum. This scenario is especially dangerous for thin walled structures that cannot be armoured due to weight penalty reasons.

Since the complete simulation of tumbling projectile impact that lead to almost spherical cavities is still an open research topic, an approach has been proposed by the authors that consists in studying this complex phenomenon by using analytical models. Fourest et al. [7] proposed an approach based on the classic Rayleigh-Plesset equation that describes the dynamics of a single gas bubble in an infinite liquid domain. Experimental HRAM bubble dynamics presented in [1] were described using a modified version of the Rayleigh-Plesset equation introducing confinement effects of a spherical container on the bubble dynamics, firstly with the calibration of the structure response [7] and secondly with the structure response being approximated using analytical plate formulae [8]. It included the effects of most of the factors identified by pioneer researchers on this subject [9-13]. These factors can be sorted out with respect to the three media: the projectile (shape, size, and material), the tank wall (thickness, material, pre-stress, and protective structure) and the liquid properties.

The objective of the approach is to predict the structure damage subsequent to an HRAM event. However in all the studies performed $[7,8,14]$ the container has been considered elastic. The 
authors believe that it has little sense to consider a more complex material behaviour in a 1D model such as Rayleigh-Plesset. Two solutions are considered to couple this approach with a finite element model for the container that would enable to deal with more complex geometries and behaviour. The first one is to directly apply the pressure field computed with Rayleigh-Plesset to the finite element model of the container. The second would be to simulate the bubble growth directly in the finite element simulation. The later one is considered in the present work. It has the advantage of more strongly coupling the bubble dynamics with the container parameters ( size, geometry,...), and it would also permit to deal with more generic cases such as non centred bubble, but it is obviously more computationally expensive.

The first step is to validate the finite element simulations in a simple case (i.e. spherical container). Due to the lack of experimental reference the simulations are compared to an analytical solution which is spherical. Compressible finite element simulation will be compared to the Keller-Miksis equation adapted by the authors to a confined situation. This model should consider enough liquid compressibility for the bubbles and containers of interest. The problem may seem to be purely academic however spherical container exists especially in the space domain or civil engineering.

\section{Formulation of the confined Keller-Miksis model}

Early studies on bubble dynamics began with Lord Rayleigh [15] who examined the pressure prediction during the collapse of a spherical bubble, assuming that the surrounding liquid is incompressible. His work was extended by Plesset [16], who derived the second-order non-linear ordinary differential equation for the time-dependent bubble radius evolution, which became the well known Rayleigh-Plesset equation for bubble dynamics. Improvements of this equation have been proposed by numerous authors, in particular Keller-Miksis [17] to include the effect of liquid compressibility on the bubble dynamics and to study the propagation of the wave emitted during the collapse.

A modification of the standard Rayleigh-Plesset equation has been proposed [7] to account for confinement effects without changing the standard Rayleigh-Plesset's resolution method. In the present section the authors propose an improvement of this model by taking liquid compressibility into account in an analytical confined bubble dynamic model.

\subsection{The Keller-Miksis model}

This model is based on the Keller-Miksis [18] model which is a modification of the Keller-Kolodner [17] model to include the effect of a convergent liquid velocity potential on the bubble dynamics. The Keller-Miksis model is presented hereafter. It is here written under the assumptions of spherical deformation of the bubble interface (no fission of the bubble), no gravity, no viscosity and surface tension effect. The bubble is at the centre of a spherical container of internal radius $R_{s}$.

The derivation of the Keller-Miksis model is detailed in Appendix. The Keller-Miksis model is based on the assumption that the velocity potential $\phi$ respects the wave equation, hence it can be written in (1) as the sum of a converging and diverging velocity potential. It is the fact that this model readily includes a converging velocity potential that is of interest for this application.

$\phi=\frac{f(t-r / c)}{r}+\frac{g(t+r / c)}{r}$ where $t$ is the time, $r$ the spacial coordinate and $c$ the speed of sound. The Keller-Miksis standard equation is:

$$
\begin{aligned}
-\ddot{R_{b}} R_{b}(\dot{R} b-c)= & \frac{1}{2}{\dot{R_{b}}}^{3}+\dot{R_{b}} \Delta\left(R_{b}\right)-c\left(\frac{3}{2} \dot{R}_{b}^{2}-\Delta\left(R_{b}\right)\right) \\
& +R_{b} \dot{R_{b}} \Delta^{\prime}\left(R_{b}\right)+2\left(1+\frac{\dot{R_{b}}}{c}\right) g^{\prime \prime}\left(t+\frac{R_{b}}{c}\right)=0
\end{aligned}
$$

where $R_{b}$ is the bubble radius, $\Delta\left(R_{b}\right)=\left(P_{b}-P_{\infty}\right) / \rho_{l}, P$ the pressure and $\rho_{l}$ the liquid density.

\subsection{Determination of $g$}

To solve this differential equation the function $g\left(t+R_{b}(t) / c\right)$ must be expressed. This function is used to account for confinement effects of a container on the bubble dynamics. First we need to calculate $f$ and $f^{\prime}$ that are expressed in (3) and (4):

$$
\begin{aligned}
f\left(t+\frac{R_{b}}{c}\right)= & -R_{b}^{2} \dot{R_{b}}+\frac{R_{b}^{2}}{c}\left(\Delta\left(R_{b}\right)+\frac{\dot{R}_{b}{ }^{2}}{2}\right)-g\left(t+\frac{R_{b}}{c}\right) \\
& +\left(\frac{2 R_{b}}{c} g^{\prime}\left(t+\frac{R_{b}}{c}\right)\right)
\end{aligned}
$$

$f^{\prime}\left(t+\frac{R_{b}}{c}\right)=-R_{b} \Delta\left(R_{b}\right)-g^{\prime}\left(t+\frac{R_{b}}{c}\right)-\frac{1}{2} R_{b} \dot{R}_{b}^{2}$

Initially $g=g^{\prime}=0$ then for $t>\left(R_{s_{0}}-R_{b_{0}}\right) / c$, which is the time needed by the initial velocity potential to reach the structure surface, $g^{\prime}$ is expressed in (5). $g$ is obtained by numerical integration of $g^{\prime}$, and $g^{\prime \prime}$ by the numerical differentiation of $g^{\prime}$.

$$
\begin{aligned}
g^{\prime}\left(t+\frac{R_{s}}{c}\right)= & R_{s} c\left(\dot{R}_{s}+\frac{1}{R_{s}^{2}}\left(f\left(t+\frac{R_{s}}{c}\right)+g\left(t+\frac{R_{s}}{c}\right)\right)\right. \\
& \left.+\frac{1}{R_{s} c} f^{\prime}\left(t+\frac{R_{s}}{c}\right)\right)
\end{aligned}
$$

The relations between $f$ and $g$ at the bubble and structure surfaces are obtained using the wave equation (1) with a constant speed of sound $c$, which leads to $f\left(t_{1}+\frac{R_{b}}{c}\right)=f\left(t_{2}+\frac{R_{s}}{c}\right)$ with $t_{2}=\left(R_{s}\left(t_{2}\right)-R b\left(t_{1}\right)\right) / c+t_{1}$ and $g\left(t_{1}+\frac{R_{s}}{c}\right)=g\left(t_{2}+\frac{R_{b}}{c}\right)$ with $t_{2}=\left(R_{s}\left(t_{1}\right)-R b\left(t_{2}\right)\right) / c+t_{1}$.

\section{Application to a rigid wall case}

In the present work it has been decided to simulate an air bubble growth that is caused by an initial gas over-pressure to validate the proposed modification of the Keller-Miksis model since it is a more classic case. The domain of liquid is at rest at the beginning of the simulation. The initial conditions are the following: $R_{b_{0}}=40 \mathrm{~mm}$, $\dot{R}_{b_{0}}=0 \mathrm{~m} . \mathrm{s}^{-1}$ and $P_{b_{0}}=6 \mathrm{MPa}$ to compare with previous reference cases [14]. These initial conditions produce bubble dynamics comparable to dynamics observed during HRAM events [7]. The first case of interest is the case of a bubble inside a spherical rigid container. In that case the velocity of the structure wall is zero, which leads to (6):

$$
\left\{\begin{array}{l}
R_{s}=R_{s_{0}} \\
\dot{R_{s}}=0
\end{array}\right.
$$

Firstly the self-consistency of the model is examined and the results obtained with the Keller-Miksis model are compared with results obtained by finite element simulations. The finite element simulations were performed using the EUROPLEXUS explicit code, which is jointly developed by CEA and EU CRC-Ispra to accurately solve fast dynamic problems for coupled fluid/structure systems. 


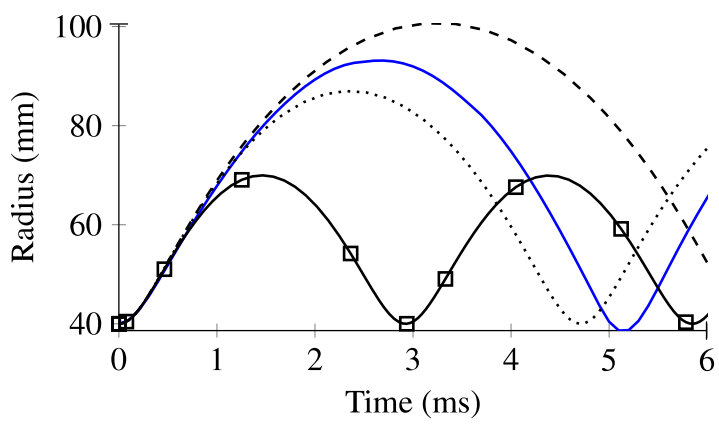

Fig. 1. Bubble radius predicted using the confined Keller-Miksis equation in a spherical rigid container (-) and predicted with the confined Rayleigh-Plesset equation in a $6 \mathrm{~mm}$ thick $(---), 15 \mathrm{~mm}$ thick (.....) and $60 \mathrm{~mm}$ thick $(\square-) R_{S}$ $=1 \mathrm{~m}$ steel container.

\subsection{Verification of the consistency of analytical models for rigid con- tainers}

The confined Keller-Miksis model is solved using an Euler method with a fixed time step $\Delta t=10^{-5} \mathrm{~ms}$. The standard Keller-Miksis equation is equivalent to the Rayleigh-Plesset equation when the liquid speed of sound tends to infinity [18]. Unfortunately this verification cannot be carried out for this case since the confined Rayleigh-Plesset equation cannot be used in a rigid container: due to the liquid incompressibility hypothesis an increase in the bubble radius can only occur simultaneously with an increase in the structure radius. However Fig. 1 shows the comparison between bubble dynamics predicted with the confined Keller-Miksis equation in a rigid $R_{s}=1 \mathrm{~m}$ spherical container and predicted using the confined Rayleigh-Plesset equation for several container rigidities. The material parameters used are summarized in Table 2. The confined Rayleigh-Plesset equation in a spherical elastic container is given by (7). For more details on the derivation of this model please refer to Fourest et al. [7].

$$
\left\{\begin{array}{l}
R_{b} \ddot{R}_{b}+\frac{3}{2}{\dot{R_{b}}}^{2}+\frac{P_{s}(t)-P_{b}(t)}{\rho}-2{\dot{R_{b}}}^{2}\left(\frac{R_{b}}{R_{s}}\right)-\ddot{R_{b}}\left(\frac{R_{b}^{2}}{R_{s}}\right) \\
+\frac{1}{2} \dot{R}_{b}^{2}\left(\frac{R_{b}}{R_{s}}\right)^{4}=0 \\
P_{s}-P_{s_{0}}=\left(R_{s}-R_{s_{0}}\right) \cdot E \cdot\left(\frac{\left(R_{s_{0}}+e\right)^{3}-R_{s_{0}}^{3}}{R_{s_{0}}^{3}}\right) \\
\times\left(\frac{2 R_{s_{0}}^{2}}{2(1-2 v) R_{s_{0}}^{3}+(1+v)\left(R_{s_{0}}+e\right)^{3}}\right)
\end{array}\right.
$$

with $E$ the Young Modulus, $v$ the Poisson ratio and $e$ the shell thickness.

The bubble predicted in the $6 \mathrm{~mm}$ thick steel spherical container using the confined Rayleigh-Plesset equation has larger oscillations than those predicted in the rigid container, however the bubbles in the $15 \mathrm{~mm}$ and $60 \mathrm{~mm}$ thick steel spherical containers have lower oscillations than in the rigid container. This is obviously not possible but it was already known that for containers with high rigidity the confined Rayleigh-Plesset equation could lead to nonphysical results. By taking the liquid compressibility into account the confined Keller-Miksis equation solves this problem.

Fig. 2 shows the bubble radius versus time for several internal radius $R_{s}$ of the spherical rigid container. As expected larger containers lead to larger bubble radius. For very large containers the predicted bubble dynamics is equivalent to the one predicted using the unconfined Keller-Miksis equation. Moreover contrary to the unconfined Keller-Miksis, the confined equation does not present a damping of the bubble amplitude at each rebound due

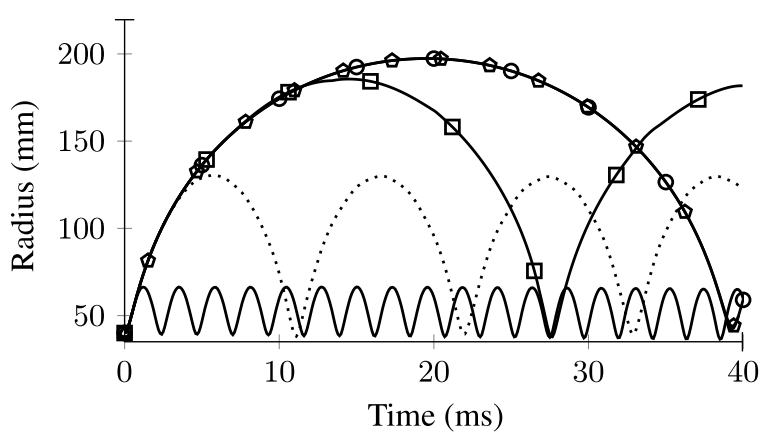

Fig. 2. Bubble radius predicted using the confined Keller-Miksis equation in several spherical rigid containers of internal radius $R_{s} . R_{S}=0.5 \mathrm{~m}(-), R_{S}=2 \mathrm{~m}(\ldots \ldots), R_{S}=$ $5 \mathrm{~m}(\bullet-), R_{S}=50 \mathrm{~m}(\neg)$ and $R_{S}=\infty$ (๑) .

to the liquid wave emitted outward. In this model this wave is eventually reflected by the structure and continues to interact with the bubble.

The Keller-Miksis model is based on the hypothesis that the velocity potential propagates at the speed of sound. Fig. 3 shows an example of the behaviour of the model for a rigid wall at $R_{\mathrm{s}}=2$ $\mathrm{m}$ of the bubble centre. The bubble radius reaches a maximal value of approximately $150 \mathrm{~mm}$. Fig. 3-(a) presents the propagation of the initial wave and its subsequent reflections on the structure and bubble walls. It can be noted that when this wave interacts with the structure surface it corresponds to changes in the pressure at this interface (see Fig. 3-(b)). As expected, the interaction between the structure and the bubble growth is effectively caught through the velocity potential propagation.

\subsection{Finite element modelling for fluid and structure in EUROPLEXUS}

Due to the geometry of the problem, uni-axial simulations should be performed. However it is not available in the EUROPLEXUS code, so axi-symmetric bi-dimensional simulations are performed. The mesh domain is reduced to a quarter with symmetry conditions being applied in the $\mathrm{x}$ and $\mathrm{y}$ directions. To strictly ensure the separation between the gas and liquid domains (see Fig. 4), an Arbitrary Lagrangian Eulerian (ALE) representation is used, where the liquid-gas interface is considered as Lagrangian. Excessive deformations within the fluid domains are avoided through classical grid motion techniques [19].

Among the several available discretization methods available in EUROPLEXUS (i.e. finite element, finite volume, SPH or discrete elements), the proposed simulations implement an hybrid formulation, with a non-conservative finite element approximation for velocities, whereas the mass conservation is achieved through a finite volume scheme. This approach allows the kinematic quantities to be located at the nodes of the mesh and provides simplicity and robustness for Arbitrary Lagrangian Euler representation. After spacial discretization, the equations to solve are the momentum conservation for the fluid (8), the dynamic equilibrium for the structure (9), the mass conservation for the fluid (10) and the kinematic constraints (11).

$M_{f} \dot{U}+\left(F_{\text {transport }}(U)+F_{\text {pressure }}(U)\right)+C_{f}^{T} \Lambda=F_{\text {ext }}$

$M_{s} \ddot{X}+F_{\text {int }}(X)+C_{f}^{T} \Lambda=F_{\text {ext }}$

$\dot{\bar{\rho}}=\Sigma_{\text {cellfaces }} \rho_{\text {upwind }} U_{\text {face }} . n s$

$C_{f} \dot{U}+C_{s} \ddot{X}=B$ 

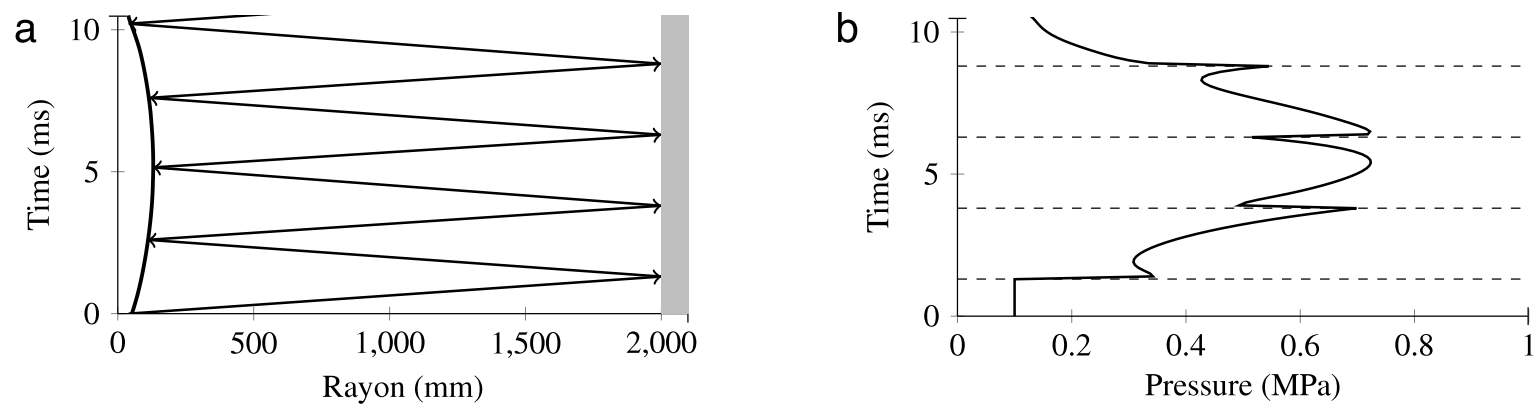

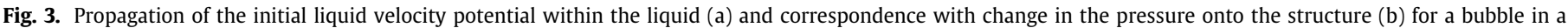
$R_{s}=2$ m rigid container

where $U$ is the fluid velocity and $X$ is the structural displacement at mesh nodes, $M_{f}$ and $M_{s}$ are the mass matrices, both made diagonal through lumping techniques, $F_{\text {transport }}$ accounts for the convective term in Euler equations, $F_{\text {pressure }}$ results from the integration of the fluid pressure forces, $F_{\text {int }}$ results from the integration of the structural stress forces, $\bar{\rho}$ is the average density within the fluid cells, $\rho_{\text {upwind }}$ and $\rho_{\text {face }}$ are the components of the simple Finite Volume scheme used to ensure mass conservation for the fluid, $C_{f}$ and $C_{s}$ express kinematic constraints acting on both fluid and structure.

The fluid is supposed to undergo adiabatic transformations, so no conservation equation for energy is required. In the next paragraphs of the current section, the structure is assumed to be rigid, so the dynamic equilibrium equation is not taken into account. It is relevant for Section 4, where the structural elasticity is introduced in the dynamic system.

Time integration is carried out through the central differences explicit scheme, writing from step $n$ to step $n+1$ (and involving mid-step quantities):

$U^{n+\frac{1}{2}}=U^{n}+\frac{\Delta t}{2} \dot{U}^{n}$

$\dot{X}^{n+\frac{1}{2}}=\dot{X}^{n}+\frac{\Delta t}{2} \ddot{X}^{n}$

$X^{n+1}=X^{n}+\Delta t \dot{X}^{n+\frac{1}{2}}$

The kinematic constraints are enforced exactly through Lagrange Multiplier $\Lambda$, with no additional arbitrary parameters such as penalty coefficients. They account for all boundary conditions, for fluid-structure interaction and for particular additional conditions (see below). Fluid-structure interaction is expressed by a linear constraint along the normal direction to the structure imposing the fluid and structural velocities to be equal in this direction (and equal to zero in the particular case of rigid structures), with the current hypothesis that the mesh nodes for fluid and structure are geometrically coincident on the interface.

Moreover, the sphericity of the Lagrangian bubble interface (free surface) is imposed in the simulations, in order to avoid unstable phenomena to occur during collapse due to a small deviation in the mesh, and hence permit the study of several bubble periods. This is achieved by imposing the velocities of all concerned fluid nodes to be equal in the radial direction, which fits directly into the algorithmic framework previously described. The numerical setup is described in Fig. 4.

\subsubsection{Material laws used in confined bubble simulations}

It is assumed that no thermal effects affect bubble dynamics in water at ambient temperature [20]. Hence the behaviour of the liquid is assumed to be isotherm. The equation of state used for the liquid is (15) with $c_{l}=1500 \mathrm{~m} . \mathrm{s}^{-1}, P_{l_{0}}=0.1 \mathrm{MPa}$ and

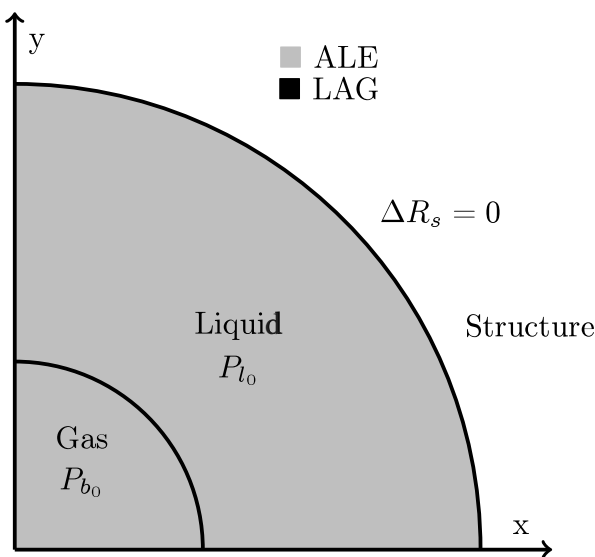

Fig. 4. Sketch of the considered problem for simulations of bubble dynamics inside a rigid container.

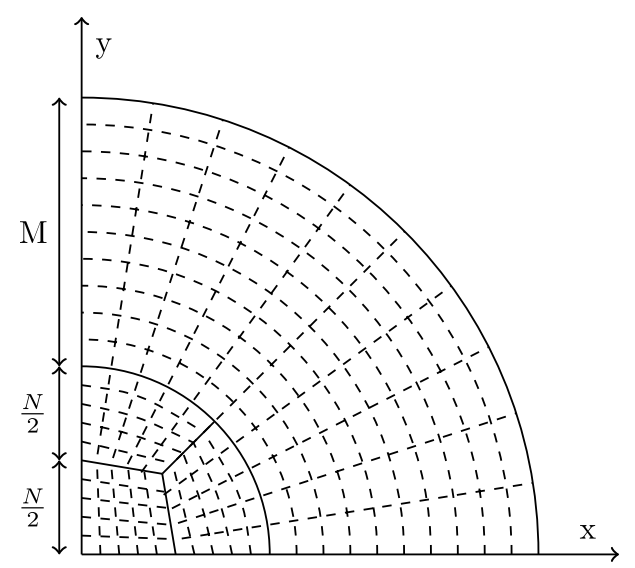

Fig. 5. Mesh principle for simulations of bubble dynamics inside a rigid container.

$\rho_{l_{0}}=1000 \mathrm{~kg} \cdot \mathrm{m}^{-3}$. The gas follows the ideal gas law (26) with $\gamma=1.4$ for air as a diatomic gas.

$P_{l}=P_{l_{0}}+\left(\rho_{l}-\rho_{l_{0}}\right) c_{l}^{2}$

\subsubsection{Convergence study for the rigid container case}

The finite element models are made of 4 nodes axi-symmetric elements. Fig. 5 shows the parameters used in the description of the mesh. $\mathrm{N}$ is the number of orthoradial elements and $\mathrm{M}$ the number of liquid elements in the radial direction. Fig. 6 shows the bubble radius versus time curves for different mesh refinements. This study is performed for $R_{S}=1 \mathrm{~m}$. 


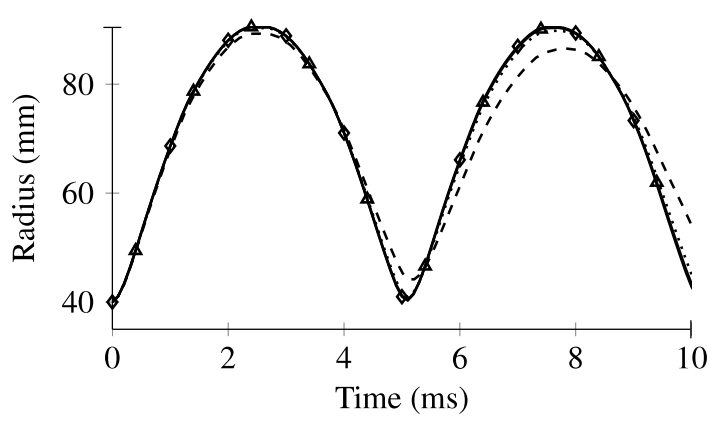

Fig. 6. Bubble radius vs time results obtained with different mesh refinements NxM: $10 \times 159(-), 24 \times 318(\neg), 56 \times 640(\multimap), 110 \times 1280(\multimap)$.

Table 1

Model features and calculation performances on Intel ${ }^{\circledR}$ Ivy-Bridge E5-2667v2 processors clocked at $3.3 \mathrm{GHz}$.

\begin{tabular}{lrrll}
\hline Model & Elements & \multicolumn{1}{c}{ Nodes } & \multicolumn{1}{c}{$\Delta t_{0}$} & CPU time \\
\hline $1 \times 158$ & 1665 & 1840 & $6.41 \cdot^{-4} \mathrm{~ms}$ & $28 \mathrm{~s}$ \\
$1 \times 318$ & 8088 & 8444 & $2.53 \cdot \cdot^{-4} \mathrm{~ms}$ & $2 \mathrm{~min} \mathrm{31 \textrm {s }}$ \\
$1 \times 640$ & 38024 & 38746 & $1.03 \cdot \cdot^{-4} \mathrm{~ms}$ & $25 \mathrm{~min}$ \\
$1 \times 1280$ & 149985 & 151432 & $5.16 \cdot \cdot^{-5} \mathrm{~ms}$ & $11 \mathrm{~h} 40 \mathrm{~min}$ \\
\hline
\end{tabular}

Table 2

Material numerical values used in the simulations.

\begin{tabular}{llll}
\hline Material & $\begin{array}{l}\rho_{s} \\
\left(\mathrm{~kg} . \mathrm{mm}^{-3}\right)\end{array}$ & \multicolumn{1}{l}{$\begin{array}{l}\text { (MPa }) \\
(\mathrm{MP}\end{array}$} & $v$ \\
\hline Steel & $7.8 \cdot 10^{-3}$ & 210000 & 0.3 \\
Aluminium & $2.7 \cdot 10^{-3}$ & 70000 & 0.3 \\
PMMA & $1.18 \cdot 10^{-3}$ & 2600 & 0.3 \\
\hline
\end{tabular}

A satisfying convergence is obtained for the $56 \times 640$ mesh refinement $(<1 \%$ of relative error with the $110 \times 1280)$. For the following analyses $N=56$ will be used. Table 1 presents the numerical cost of each finite element simulation for $10 \mathrm{~ms}$ of simulated time. The computational time for the Keller-Miksis simulations is approximately of $5 \mathrm{~s}$.

\subsection{Comparison of the confined Keller-Miksis and finite element simulations for bubbles in rigid containers}

The results obtained with the confined Keller-Miksis model in a rigid container are compared to those obtained by finite element simulations. Fig. 7 compares the radius versus time evolution for a bubble in three containers of internal radius $R_{s}=0.5 \mathrm{~m}, 1 \mathrm{~m}$ and $2 \mathrm{~m}$. Fig. 8 compares the pressure at the bubble and structure surfaces calculated with the confined Keller-Miksis and finite element model for a $R_{S}=1 \mathrm{~m}$ rigid container. The results obtained in all cases are in a fairly good agreement. The radii predicted with the finite element models were found to be slightly inferior in terms of amplitude and period duration to the one predicted with the confined Keller-Miksis model. The same tendency is observed for the predicted pressure, in particular the pressure in the bubble obtained with the finite element model during the first rebound is inferior to prediction of the analytical model. It is attributed to the fact that the analytical model does not consider hydrodynamic decay (compression and tension wave propagate at the same velocity). Nevertheless the two models are in very good agreement which validates the analytical model developed and the finite element simulations used.

\section{Application to an elastic wall case}

Now that the case of a rigid container has been validated, the case of bubbles in an elastic container is examined. Instead of (7)

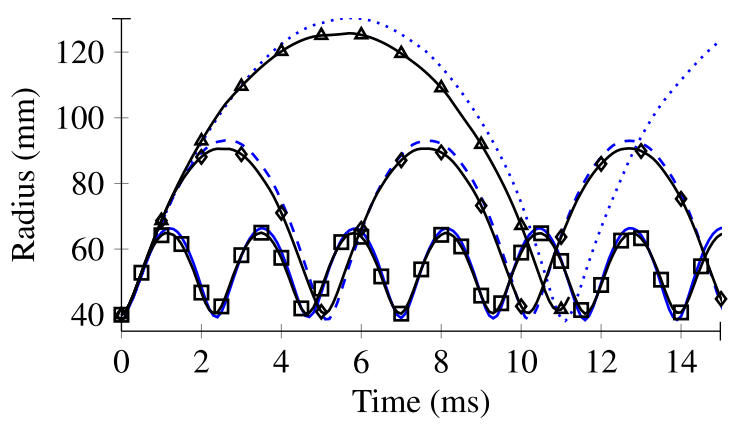

Fig. 7. Bubble radius predicted using the confined Keller-Miksis equation in a $R_{s}=0.5 \mathrm{~m} \mathrm{(-)}, R_{s}=1 \mathrm{~m} \mathrm{(--)}$ and $R_{s}=2 \mathrm{~m}(\ldots$.$) rigid containers and$ obtained by ALE finite element simulations in $R_{S}=0.5 \mathrm{~m}(\square-), R_{S}=1 \mathrm{~m} \mathrm{( \hookrightarrow -)}$ and $R_{s}=2 \mathrm{~m}(\neg)$ spherical rigid containers.

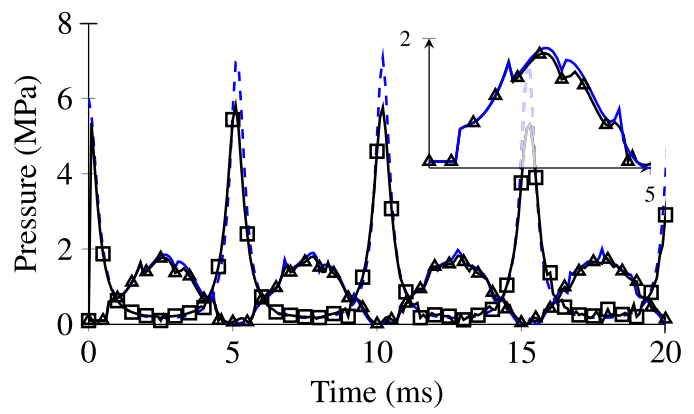

Fig. 8. Pressure at the bubble and structure interfaces predicted using Keller-Miksis equation (_- ) and (- - -) and obtained by ALE finite element bubble growth simulation $(\square-)$ and $(\longrightarrow-$ ).

that models the static response of a spherical shell submitted to uniform internal pressure, a dynamic response model is used for the spherical structure. This is done to ensure the continuity of the function $R_{s}$ that is obtained by numerical integration. For reasons of simplification the internal radius of the sphere is used as the mean surface of the shell. It is justified since the shell thickness is largely inferior to the shell internal radius.

In the case of a spherical shell the curvature is constant and displacement only occurs in the radial direction. The strains are calculated using Reissner's polynomial approximation [21] (16).

$\varepsilon_{\theta \theta}=\varepsilon_{1_{\theta \theta}}+\varepsilon_{2_{\theta \theta}}\left(r-R_{S}\right)+\varepsilon_{3_{\theta \theta}}\left(r-R_{S}\right)^{2}$

with $\varepsilon_{\theta \theta}$ the membrane coefficient, $\alpha_{\theta \theta}$ the bending coefficient and $\beta_{\theta \theta}$ the quadratic coefficient, given in the case of a spherical shell in (17),

$\varepsilon_{1_{\theta \theta}}=\frac{R_{s}-R_{S_{0}}}{R_{s_{0}}} ; \varepsilon_{2_{\theta \theta}}=-\frac{R_{s}-R_{s_{0}}}{R_{s_{0}}{ }^{2}} ; \varepsilon_{3_{\theta \theta}}=\frac{R_{s}-R_{s_{0}}}{R_{s_{0}}{ }^{3}}$

Using Hooke's law the following stresses are found in (18),

$$
\begin{aligned}
\sigma_{\theta \theta}=E \frac{1+v}{1-v^{2}} \varepsilon_{\theta \theta}= & E \frac{1+v}{1-v^{2}}\left(R_{s}-R_{s_{0}}\right) \\
& \times\left(\frac{1}{R_{s_{0}}}-\frac{\left(r-R_{s}\right)}{R_{s_{0}}^{2}}+\frac{\left(r-R_{s}\right)^{2}}{R_{s_{0}}^{3}}\right)
\end{aligned}
$$

Then the membrane flux $N_{\theta \theta}$ can be calculated using (19):

$N_{\theta \theta}=\frac{E(1+v)}{1-v^{2}}\left(e \varepsilon_{1_{\theta \theta}}+\frac{e^{3}}{12}\left(\varepsilon_{3_{\theta \theta}}+\frac{\varepsilon_{2_{\theta \theta}}}{R_{s_{0}}}\right)\right)$

Finally, using the equilibrium equation (20) it is possible to obtain the equation of motion of a spherical shell submitted to spatially 


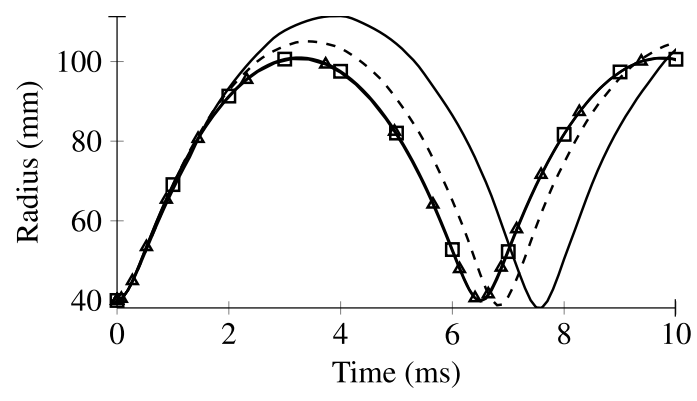

Fig. 9. Bubble radius vs. time in a $R_{s}=1 \mathrm{~m} 6 \mathrm{~mm}$ thick steel spherical container predicted with the confined Keller-Miksis equation with $c_{l}=1500 \mathrm{~m} . \mathrm{s}^{-1}(-)$, $c_{l}=2500 \mathrm{~m} \cdot \mathrm{s}^{-1}(---), c_{l}=10000 \mathrm{~m} \cdot \mathrm{s}^{-1}(\square-)$ and the confined RayleighPlesset equation with $c_{l}=\infty\left(\longrightarrow \_\right)$.

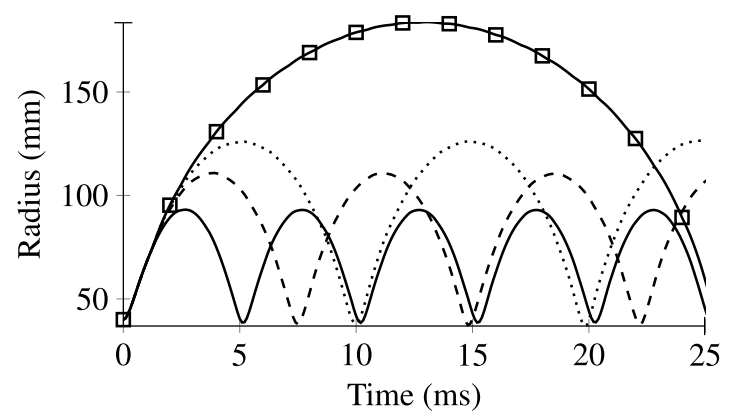

Fig. 10. Bubble radius vs. time predicted with the confined Keller-Miksis equation in $R_{s}=1 \mathrm{~m}$ rigid $(-), 6 \mathrm{~mm}$ thick steel $(---)$, aluminium $(\ldots \ldots)$, PMMA (—-) spherical containers.

uniform pressure (21) which permits to calculate $\dot{R}_{s}$ and $R_{s}$.

$$
\begin{aligned}
& \frac{-2 N_{\theta \theta}}{R_{s_{0}}}+P_{s}-P_{s_{0}}+\rho h \ddot{R}_{s}=0 \\
& -\rho_{s} e \ddot{R}_{s}+P_{s}-P_{s_{0}}-\frac{2 E(1+v)}{1-v^{2}} \frac{\left(R_{s}-R_{s_{0}}\right)}{R_{s_{0}}^{2}}\left(e+\frac{2 e^{3}}{12 R_{s_{0}}}\right)=0
\end{aligned}
$$

The fluid and structure equations are coupled using (22) to calculate the term $P_{S}$ in (21).

$P(r, t)=P_{\infty}-\rho_{l}\left(\frac{\partial \phi}{\partial t}+\frac{1}{2}\left(\frac{\partial \phi}{\partial r}\right)^{2}\right)$

\subsection{Verification of the consistency of analytical models for elastic} containers

The classic Keller-Miksis equation is equivalent to the Rayleigh-Plesset equation when the liquid speed of sound tends to infinity [18]. Therefore it is first verified that when the speed of sound in the liquid is chosen high, both confined models predict the same bubble radius evolution. Fig. 9 compares the bubble radius predicted in a $6 \mathrm{~mm}$ thick steel spherical container with the KellerMiksis for several values of the sound speed to the radius predicted in the same container using the confined Rayleigh-Plesset equation. It can be observed that by increasing the sound speed in the liquid the confined Keller-Miksis converges to the confined Rayleigh-Plesset model. For a sound speed of $c_{l}=10000 \mathrm{~m} \cdot \mathrm{s}^{-1}$ the results obtained using both models are virtually identical.

The development of confined bubble dynamics models is carried out to take the interaction between the bubble and the structure into account. Fig. 10 compares the predicted bubble dynamics in a rigid container and in several containers of different overall rigidity. The model behaves in the expected way, the bubble amplitude and period increase while the overall rigidity of the container decreases.

Fig. 11 shows an example of the behaviour of the model for a $6 \mathrm{~mm}$ thick steel wall at $R_{s}=2 \mathrm{~m}$ of the centre of the bubble for one period of the bubble. Fig. 11-(a) recalls the propagation of the initial wave and its subsequent reflections on the structure and bubble walls. Fig. 11-(b) is a zoom of Fig. 11-(a) at the deformable structure surface. It can be noticed that the times at which the wave interacts with the structure surface correspond to changes in the pressure at this interface that can be seen in Fig. 11-(c). The pressure observed in this case differs from the one in Fig. 3-(b): there is a quick increase of the pressure at times when the initial wave interacts with the structure wall. However this increase is rapidly compensated by the structure deformation, hence smaller discontinuities in the pressure response are observed in this case. As expected the pressure found at the container surface in this elastic case is less than the one obtained for the same source in a rigid container in Fig. 3-(b). It can also be noted that at the end of the period neither the structure wall position nor the pressure applied to it reach the initial value.

\subsection{Comparison of the confined Keller-Miksis and finite element simulations for bubbles in elastic containers}

\subsubsection{Finite element modelling of the elastic case}

The problem is here the same as in Section 3.2 except that an external spherical structure has been added. The bubble interface (between liquid and air) and the structure nodes displacements are again defined Lagrangian and spherical. So there is no mixture between the air and water in the simulations. Fig. 12 describes the considered problem.

\subsubsection{Material laws used in elastically confined bubble simulations}

The liquid and gas are assumed to follow respective equations of state (15) and (26). The containers are modelled using a linear elastic law. Table 2 summarizes the different material values used for the simulations.

\subsubsection{Convergence study for bubble dynamics in an elastic container}

Again, the finite element models consist of 4 nodes axisymmetric elements. Fig. 13 shows the parameters used in the description of the mesh. $\mathrm{N}$ is the number of orthoradial elements, $\mathrm{M}$ the number of liquid elements in the radial direction and $\mathrm{L}$ the number of container elements in the radial direction. A convergence study has been performed for a bubble in a $R_{S}=1 \mathrm{~m}$ $6 \mathrm{~mm}$ thick steel container. The conclusions are the same than in Section 3.2.2, therefore the details are not presented here. Satisfying convergence is obtained for the $56 \times 640 \times 4$ mesh refinement (less than $1 \%$ of relative error with the $110 \times 1280 \times 8$ ). Therefore, the $56 \times 640 \times 4$ models will be used for the following analyses.

\subsubsection{Simulation results}

The results obtained with the confined Keller-Miksis model in elastic containers are compared to those obtained with finite element simulations. Fig. 14-(a) compares the radius versus time evolution for a bubble in three elastic containers of internal radius $R_{S}=1 \mathrm{~m}$. Fig. 14-(b) compares the predicted internal radius of the spherical structure in the same cases. The results obtained in all cases are in a fairly good agreement during the first period of bubble pulsation. The maximum difference in bubble dynamics is obtained for the bubble in the PMMA container (1\% in bubble amplitude and $3.5 \%$ in bubble period). The same order of deviation is found for the structure response. Contrary to results that are usually obtained with the confined Rayleigh-Plesset equation 

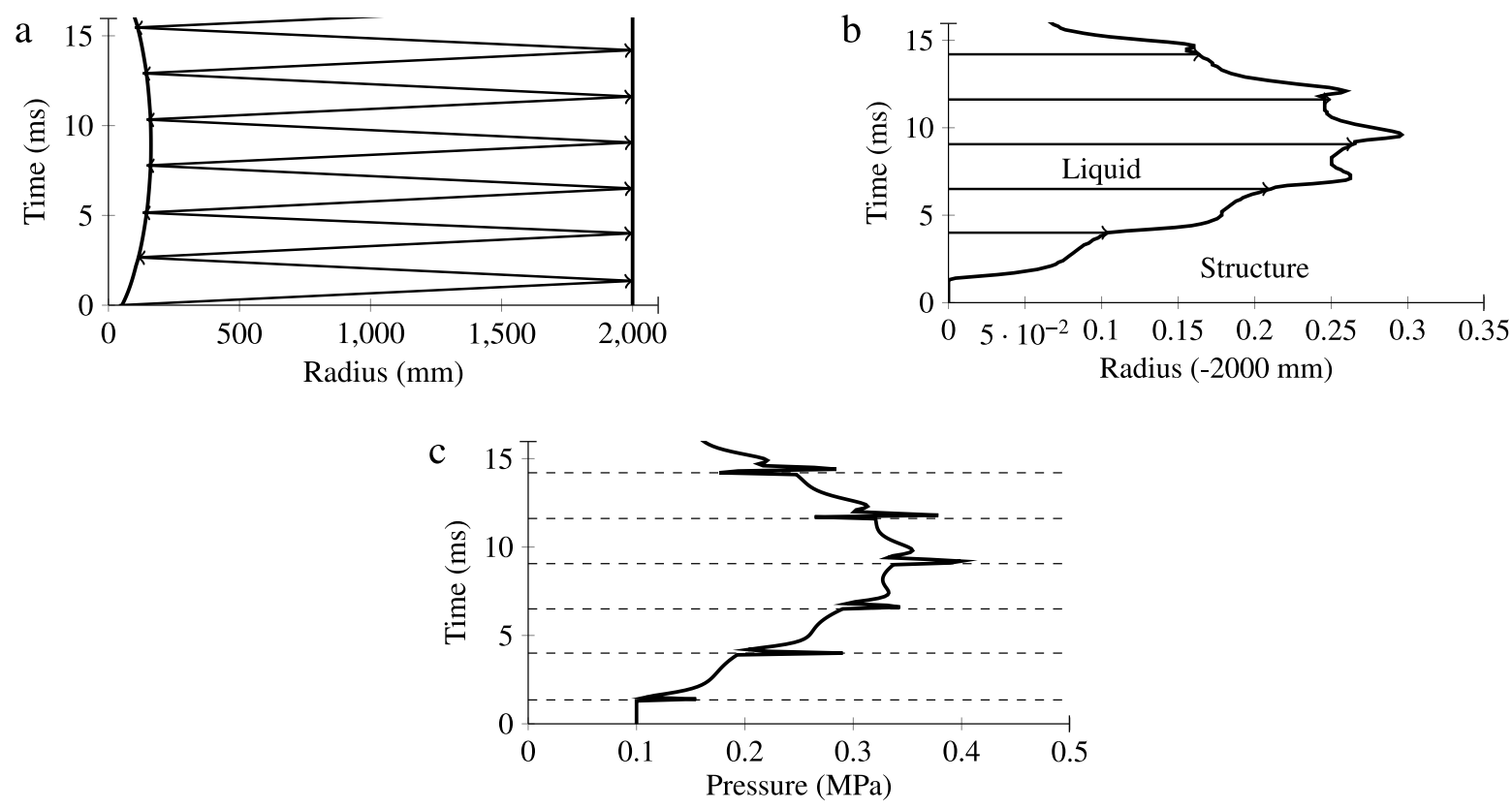

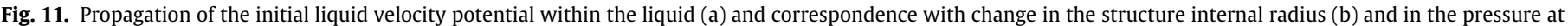
the structure wall (c) for a $R_{\mathrm{s}}=2 \mathrm{~m} 6 \mathrm{~mm}$ thick steel container.

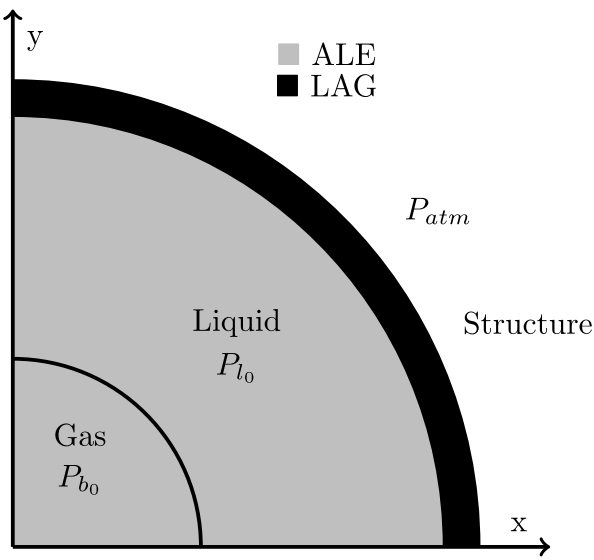

Fig. 12. Sketch of the considered problem for bubbles dynamics simulations in an elastic containers.

the response of the structure (which has a smooth movement in Rayleigh-Plesset simulations) found using the confined KellerMiksis equation oscillates in a similar manner than the one calculated using finite element simulations. Figs. 14-(c) and (d) compares the pressure at the structure and bubble surfaces calculated using the confined Keller-Miksis and finite element model for $R_{S}$ $=1 \mathrm{~m}$ elastic containers. The pressure predicted on the structure surface is in good agreement for both cases especially during the first bubble period. As in Fig. 8 the bubble pressure predicted using the Keller-Miksis model is higher during the rebound than the one predicted using finite element simulations. This is due to the fact that lower bubble radii are reached during collapse in the Keller-Miksis model possibly due to small damping in the finite element simulations. Nevertheless the two models are in very good agreement which validates again both the analytical model and finite element simulations for bubbles in elastic containers.

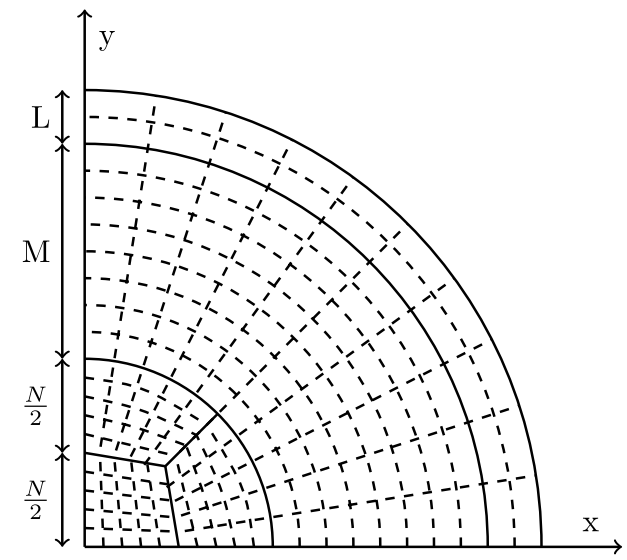

Fig. 13. Mesh principle for bubble dynamics simulations in an elastic containers.

4.3. Evaluation of the improvement in prediction by modelling the liquid compressibility

In this section the improvement obtained when modelling the liquid compressibility instead of the confined Rayleigh-Plesset equation is examined. It has already been shown that for very rigid containers the confined Rayleigh-Plesset equation can lead to non-physical results. Therefore only one comparison on a not too rigid case will be presented hereafter to illustrate the obtained improvement in the bubble dynamics, structure movement and hydrodynamic load predictions using the confined Keller-Miksis model instead of the Rayleigh-Plesset equation. Fig. 15 compares the bubble dynamics and pressure on the structure for a bubble in an elastic spherical container. The confined Rayleigh-Plesset equation predicts lower bubble radius which is logical since the liquid compressibility is expected to allow a larger bubble radius for the same pressure level. The finite element simulation and the Keller-Miksis model are quite similar in terms of bubble dynamics 

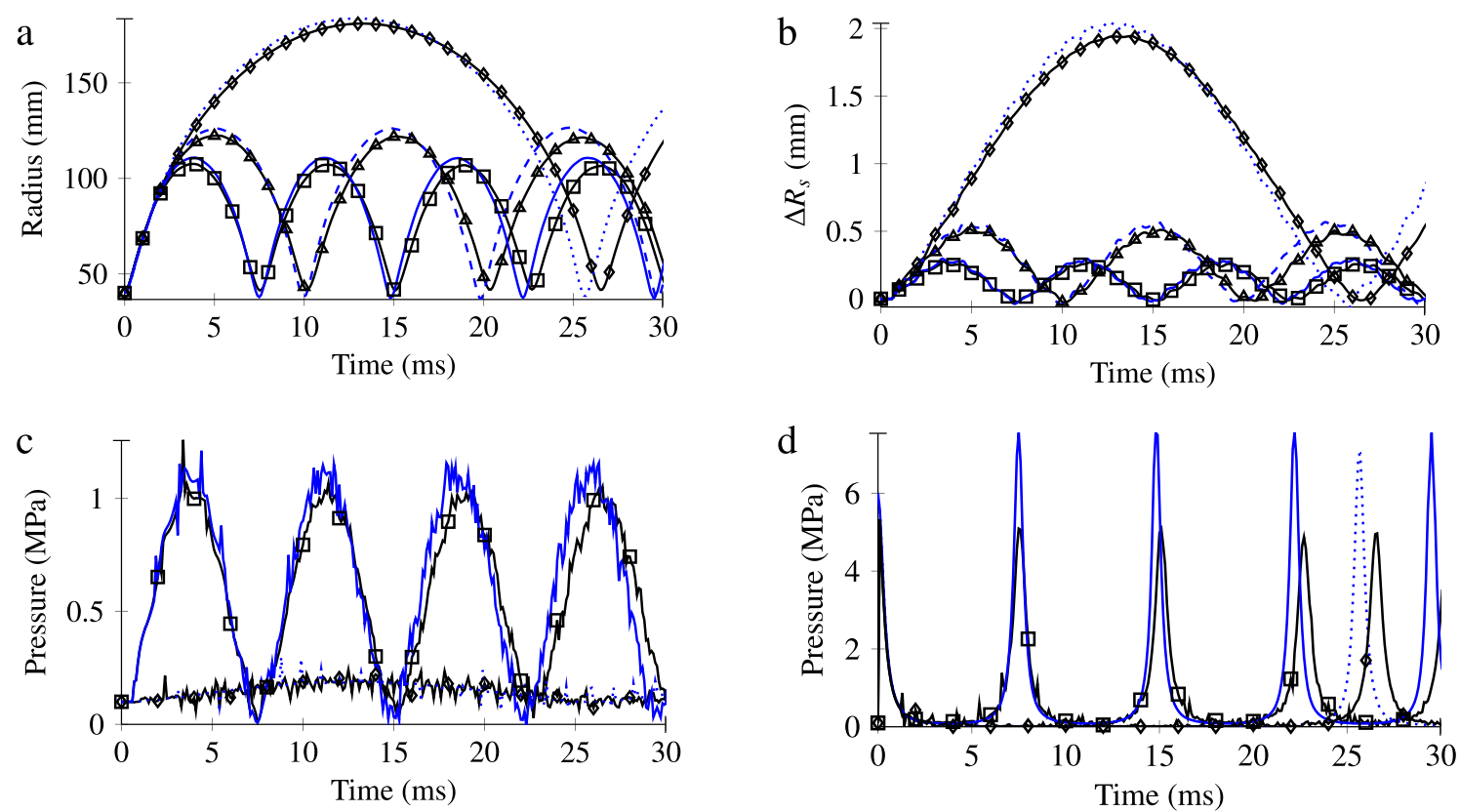

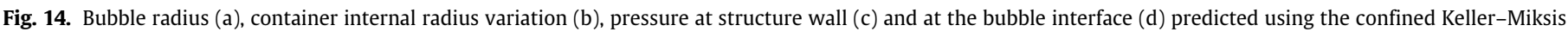

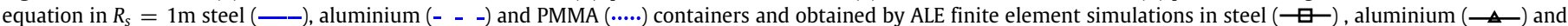
PMMA $(\neg-)$ containers.
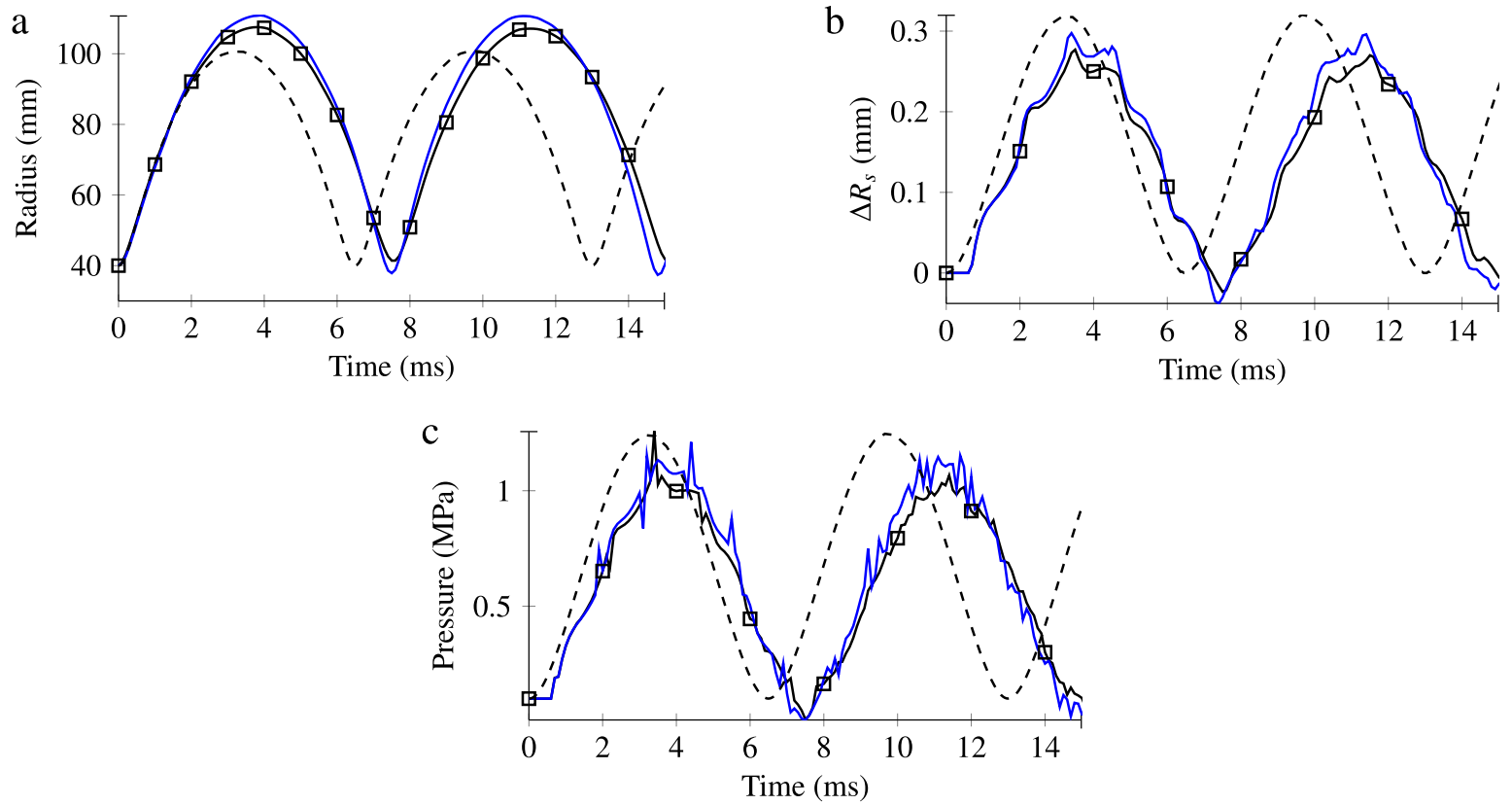

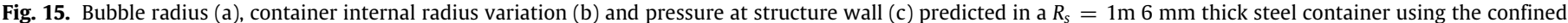
Keller-Miksis equation (_-), the confined Rayleigh-Plesset equation (- - - ) and obtained by ALE finite element simulations ( $\square-)$.

(3\% instead of $10 \%$ in amplitude and less than $1 \%$ instead of $7 \%$ in period). Similarly the pressure and internal structure radius curves predicted using the Keller-Miksis equation have a very similar shape than those obtained by finite element simulations, which is not the case for Rayleigh-Plesset. The difference in the impulsion transmitted to the structure during the first oscillation is lower (less than $1 \%$ instead of $3.5 \%$ with the confined RayleighPlesset equation). This difference would of course increase if more compressible fluids $\left(c_{l} \approx 1300 \mathrm{~m} \cdot \mathrm{s}^{-1}\right.$ for kerosene [22]) were examined. Therefore, modelling the bubble dynamics in the finite element simulation would improve the results with respect to applying the Rayleigh-Plesset pressure directly to a finite element model of the structure even in the case of a spherical container.

\section{Discussion}

In the present study the Keller-Miksis [18] equation has been used to predict the dynamics of gas bubbles in elastic spherical containers. It is a logical use for this model, even if this equation was derived by considering an infinite domain of liquid and is mostly used in that case: it was initially developed to predict 
the response of a bubble submitted to an harmonic solicitation, typically a gas bubble in a vibrating vessel. In the present study the model is used for its initial application but instead of assuming the form of the harmonic pressure field in the liquid and deducing the converging velocity potential from this assumption, the converging velocity potential is found by considering the response of the structure. In the present study two cases have been studied: rigid and elastic containers on which the only load applied is derived from the bubble dynamics. However it is also possible to apply this equation for the initial purpose by considering a structure wall having an imposed harmonic movement. It would then require no assumption on the liquid pressure profile, but only on the frequency and amplitude of the structure vibrations.

In all calculations performed in the present study the liquid has been considered inviscid due to the large dimensions of the bubbles considered in the calculation. However the liquid viscosity and surface tension may be added by modifying the boundary condition at the bubble wall. Moreover the Keller-Miksis model uses an acoustic approximation and is expected to be all the more accurate as all liquid velocities are small compared to the velocity of sound in the liquid. However this approximation is found to be in good agreement with the numerical data. The predictions of this model might still be improved by changing this approximation to an extrapolation of the acoustic theory: the Kirkwood-Bethe hypothesis (summarized in Cole [23]). It assumes that all quantities propagate at the speed $\dot{r}+c$ instead of $c$. This hypothesis is used in the Gilmore [24] model in which it is found to be accurate for bubble wall velocities up to 2.2 times the sonic velocities for which the acoustic approximation seems unlikely to produce accurate results. Hence improvement in the bubble prediction for high growing rate bubbles may be obtained by using the KirkwoodBethe equation in a similar model than the one proposed in the present study.

\section{Conclusion and outlooks}

In the present study the classic Keller-Miksis equation that models the dynamics of a single gas bubble in an infinite domain of liquid assuming a constant liquid speed of sound has been used to take the confinement effects of a spherical container on the bubble dynamics into account. To this end the function $g$ that represents the convergent part of the velocity potential in the liquid has been used. A limit condition has been written in terms of velocity potential to link this function to the divergent potential that reaches the structure. This model has been developed for two cases of containers: rigid and elastic containers. In both cases the consistency of the analytical model has been assessed, and it has been used to validate ALE finite element simulation.

Concerning the consistency of the analytical model it has been verified that:

- Contrary to the confined Rayleigh-Plesset model it does not predict non-physical results for high rigidity (or rigid) containers;

- The predicted bubble radius and period increase with the container size until it reaches the unconfined Keller-Miksis results for large containers;

- In an elastic container the confined Keller-Miksis model is equivalent to the confined Rayleigh-Plesset one for high sound speed (10000 m.s ${ }^{-1}$ );

- The predicted bubble radius and period increase while the overall rigidity of the container decreases.
In all studied cases a very good agreement has been found between the results obtained using the confined Keller-Miksis model and finite element simulations. The maximum difference in bubble dynamics is obtained for the bubble in the PMMA container ( $5 \%$ in bubble amplitude and 3.5\% in bubble period). A good agreement in the pressure prediction is found, curves predicted with both methods have the same shape and variations on the pressure have very little effect on the impulse transmitted to the structure (less than 1\%). These good agreements validate the use of finite element simulation to predict the bubble and structure dynamics. The Keller-Miksis model is largely less computationally expensive (5 s against 25 min with finite element simulation) however it is limited to spherical containers.

Finally the improvement in bubble dynamics and hydrodynamic load predictions achieved by modelling liquid compressibility has been examined by comparing the confined Keller-Miksis to the confined Rayleigh-Plesset equation and to results obtained by finite element simulations. The Keller-Miksis model predicts a bubble radius closer to the results obtained with finite element simulations that the Rayleigh-Plesset one (3\% instead of $10 \%$ in amplitude and less than $1 \%$ instead of $7 \%$ in period). Moreover the difference in the impulsion given to the structure during the first oscillation has been found to be lower (less than $1 \%$ instead of $3.5 \%$ ). However the obtained predictions with the confined RayleighPlesset stay reasonably accurate in this case. Moreover in terms of structure sizing the Rayleigh-Plesset is conservative since its predicts a slightly higher pressure and structure movement. Since the agreement between the finite element simulations and the analytical model is found very acceptable, further studies will be carried out to simulate more complex container geometries and behaviours in finite element simulations.

\section{Acknowledgements}

This study has been carried out as part of the Ph.D. thesis of the main author. The authors would like to thank the French Ministry of Defence and DGA (French Armament Procurement Directorate), for their financial support. The authors would also like to thank Dr. Daniel Fuster from the CNRS/UPMC Université Paris 06, UMR 7190 for his very valuable suggestions on the method to solve the analytical problem.

\section{Appendix. Formulation of the Keller-Miksis model}

\section{A.1. Basic fluid mechanics equations}

The velocity of the bubble interface must be equal to the velocity of the fluid at the bubble surface, which gives (23),

$\dot{R_{b}}=\frac{\partial \phi\left(R_{b}, t\right)}{\partial r}$

The velocity potential $\phi$, the pressure $\mathrm{P}$, and the liquid density $\rho_{l}$ must satisfy the equations of conservation of mass (24), the Euler equation (25) and the equations of states (26) (here the perfect gas law),

$$
\begin{gathered}
\frac{\partial \rho_{l}}{\partial t}+\frac{\partial \phi}{\partial r} \frac{\partial \rho_{l}}{\partial r}+\rho_{l}\left(\frac{\partial^{2} \phi}{\partial r^{2}}+\frac{2}{r} \frac{\partial \phi}{\partial r}\right)=0 \\
\rho\left(\frac{\partial}{\partial t} \frac{\partial \phi}{\partial r}+\frac{\partial \phi}{\partial r} \frac{\partial^{2} \phi}{\partial r^{2}}\right)+\frac{\partial P}{\partial r}=0
\end{gathered}
$$


$P_{b}=P_{b_{0}}\left(\frac{R_{b_{0}}}{R_{b}}\right)^{3 \gamma}$

\section{A.2. Simplifications introduced in the Keller-Miksis model}

The Navier-Stokes equation (25) is integrated with respect to $r$ from $r$ to infinity, which gives (27),

$$
-\frac{\partial \phi}{\partial t}-\frac{1}{2}\left(\frac{\partial \phi}{\partial r}\right)^{2}+\int_{r}^{\infty} \frac{d P}{\rho_{l}}=0
$$

Next (27) is differentiated with respect to $t$ with $\frac{\partial P}{\partial t}=\frac{d P}{\partial \rho_{l}} \frac{\partial \rho_{l}}{\partial t}=$ $c^{2} \frac{\partial \rho_{l}}{\partial t}$ where $c^{2}=\frac{\partial P}{\partial \rho_{l}}$ and $c$ is the speed of sound.

$\frac{\partial^{2} \phi}{\partial t^{2}}+\frac{\partial \phi}{\partial r} \frac{\partial^{2} \phi}{\partial t \partial r}+\frac{c^{2}}{\rho_{l}} \frac{\partial \rho_{l}}{\partial t}=0$

Then (24) is used in (28) to eliminate $\partial \rho_{l} / \partial t$, and divided by $c^{2}$ to obtain (29),

$\frac{1}{c^{2}} \frac{\partial^{2} \phi}{\partial t^{2}}-\frac{\partial^{2} \phi}{\partial r^{2}}-\frac{2}{r} \frac{\partial \phi}{\partial r}=\frac{1}{\rho} \frac{\partial \rho_{l}}{\partial r} \frac{\partial \phi}{\partial r}-\frac{1}{c^{2}} \frac{\partial \phi}{\partial r} \frac{\partial^{2} \phi}{\partial t^{2}}$

The Keller-Miksis equation is written under the assumption that for a nearly incompressible fluid $c^{2}$ is large and $\partial \rho / \partial r$ is small and that $\partial \phi \partial r$ and $\partial^{2} \phi \partial t^{2}$ have finite values. It leads to the classic wave propagation equation (30). The Keller-Miksis equation therefore consider liquid compressibility assuming a constant sound speed.

$\frac{1}{c^{2}} \frac{\partial^{2} \phi}{\partial t^{2}}-\frac{\partial^{2} \phi}{\partial r^{2}}-\frac{2}{r} \frac{\partial \phi}{\partial r}=0$

For simplification $\rho_{l}$ is set constant in (27) which gives the classic unsteady Bernoulli equation (31) for the expression of pressure in the liquid:

$P(r, t)=P_{\infty}-\rho_{l}\left(\frac{\partial \phi}{\partial t}+\frac{1}{2}\left(\frac{\partial \phi}{\partial r}\right)^{2}\right)$

\section{A.3. Derivation of the Keller-Miksis differential equation} by $\rho_{l}$.

$\Delta\left(R_{b}\right)$ is defined in (32) as the pressure difference divided

$\Delta\left(R_{b}\right)=\frac{P_{b}-P_{\infty}}{\rho_{l}}=-\frac{\partial \phi}{\partial t}-\frac{1}{2}\left(\frac{\partial \phi}{\partial r}\right)^{2}$

In the Keller-Miksis model $\phi$ respects the wave equation (30), hence it can be written in (33) as the sum of a converging and diverging velocity potential.

$\phi=\frac{f(t-r / c)}{r}+\frac{g(t+r / c)}{r}$

Subsequently equation (33) is used in (32) and in (23) to eliminate the convergent part f, it gives (34) for $R_{b}$ where $f$ does not appears [18],

$R_{b} \Delta\left(R_{b}\right)-c R_{b} \dot{R_{b}}=c \phi\left(R_{b}, t\right)-\frac{1}{2} R_{b} \dot{R}_{b}{ }^{2}-2 \dot{g}\left(t+\frac{R_{b}}{c}\right)$

Finally (34) is differentiated with respect to time and (32) is used to eliminate $\partial \phi / \partial t$, which leads to the Keller-Miksis standard equation (35).

$$
\begin{array}{r}
-\ddot{R}_{b} R_{b}(\dot{R} b-c)=\frac{1}{2}{\dot{R_{b}}}^{3}+\dot{R_{b}} \Delta\left(R_{b}\right)-c\left(\frac{3}{2} \dot{R}_{b}{ }^{2}-\Delta\left(R_{b}\right)\right) \\
+R_{b} \dot{R_{b}} \Delta^{\prime}\left(R_{b}\right)+2\left(1+\frac{\dot{R_{b}}}{c}\right) g^{\prime \prime}\left(t+\frac{R_{b}}{c}\right)=0
\end{array}
$$

\section{References}

[1] E. Deletombe, J. Fabis, J. Dupas, J.M. Mortier, Experimental analysis of $7.62 \mathrm{~mm}$ hydrodynamic ram in containers, J. Fluids Struct. 37 (2013) 1-21.

[2] P.J. Disimile, L.A. Swanson, N. Toy, The hydrodynamic ram pressure generated by spherical projectiles, Int. J. Impact Eng. 36 (6) (2009) 821-829.

[3] N. Lecysyn, A. Bony-Dandrieux, L. Aprin, F. Heymes, P. Slangen, G. Dusserre, L. Munier, C. Le Gallic, Experimental study of hydraulic ram effects on a liquid storage tank: Analysis of overpressure and cavitation induced by a high-speed projectile, J. Hazardous Mater. 178 (1) (2010) 635-643.

[4] M.S. Plesset, R.B. Chapman, Collapse of an initially spherical vapor cavity in the neighbourhood of a solid boundary, J. Fluid Mech. 47 (1971) 283-290.

[5] W. Lauterborn, H. Bolle, Experimental investigations of cavitation-bubble collapse in the neighbourhood of a solid boundary, J. Fluid Mech. 72 (2) (1975) 391-399.

[6] G.L. Chahine, R. Duraiswami, Boundary element method for calculating 2D and 3-D underwater explosion bubble behaviour in free water and near structures, in: Technical Report NSWCDD/TR-83/44, NSWC Weapons Research an Technology Department, 1994.

[7] T. Fourest, J.-M. Laurens, E. Deletombe, J. Dupas, M. Arrigoni, Analysis of bubbles dynamics created by hydrodynamic ram in confined geometries using the rayleigh-plesset equation, Int. J. Impact Eng. 73 (2014) 66-74.

[8] T. Fourest, J.-M. Laurens, E. Deletombe, J. Dupas, M. Arrigoni, Confined rayleigh-plesset equation for hydrodynamic ram analysis in thin-walled containers under ballistic impacts, Thin-Walled Struct. 86 (2015) 67-72.

[9] C.R. Morse, F.S. Stepka, Effect of projectile size and material on impact fracture of walls of liquid-filled tanks, in: Technical Report TN D-3627, NASA, Cleveland, Ohio, USA, 1966.

[10] F.S. Stepka, C.R. Morse, Preliminary investigation of catastrophic fracture of liquid-filled tanks impacted by high velocity particles, in: Technical Report D1537, NASA, Cleveland, Ohio, USA, 1963.

[11] F.S. Stepka, R.P. Dengler, C.R. Morse, Investigation of catastrophic fracturing and chemical reactivity of liquid-filled tanks when impacted by projectiles of high velocity, in: Technical Report TM X-52063, NASA, Cleveland, Ohio, USA, 1964.

[12] F.S. Stepka, C.R. Morse, R.P. DengZey, Investigation of the characteristics of pressure waves generated in water filled tanks impactes by high-velocity projectiles, in: Technical Report TN D-3143, NASA, Cleveland, Ohio, USA, 1965.

[13] F.S. Stepka, Projectile-impact-induced fracture of liquid-filled, filament-reinfo rced plastic or aluminium tanks, in: Technical Report TN D-3456, NASA, Cleveland, Ohio, USA, 1966.

[14] T. Fourest, J.-M. Laurens, E. Deletombe, M. Arrigoni, J. Dupas, Cross validation of analytical and finite element models of hydrodynamic ram loads prediction in liquid filled thin-walled containers, J. Fluids Struct. 59 (2015) 285-296.

[15] Lord Rayleigh, On the pressure developed in a liquid during the collapse of a spherical cavity, London Edinburgh Dublin Phil. Mag. J. Sci. 34 (200) (1917) 94-98.

[16] M.S. Plesset, The dynamics of cavitation bubbles, J. Appl. Mech. 16 (1949) 277282.

[17] J.B. Keller, I.I. Kolodner, Damping of underwater explosion bubble oscillations, J. Appl. Phys. 27 (10) (1956).

[18] J.B. Keller, M. Miksis, Bubble oscillations of large amplitude, J. Acoust. Soc. Am. 68 (1980) 628-633.

[19] M. Souli, A. Ouahsine, L. Lewin, Ale formulation for fluid-structure interaction problems, Comput. Methods Appl. Mech. Engrg. 190 (5-7) (2000) 659-675.

[20] C.E. Brennen, Cavitation and Bubble Dynamics, Oxford University Press, Oxford, United Kingdom, 1995.

[21] E. Reissner, On the theory of bending of elastic plates, J. Math. Phys. 23 (184) (1944)

[22] J. Aldred, Manual of Sound Recording, Fountain Press, London, 1972.

[23] R.H. Cole, Underwater Explosions, Princeton University Press, Princeton, 1945

[24] F.R. Gilmore, The growth or collapse of a spherical bubble in viscous compressible liquid, in: Technical Report 26-4, California Institute of Technology, 1952. 